\title{
Surface plasmon-related resonances on deep and asymmetric gold gratings
}

\author{
M. Kreiter, S. Mittler, and W. Knoll \\ Max Planck Institut für Polymerforschung, Ackermannweg 10, D-55128 Mainz, Germany \\ J. R. Sambles \\ Department of Physics, University of Exeter, Exeter, Devon EX4 4QL, England
}

(Received 3 May 2001; published 13 March 2002)

\begin{abstract}
Based on theoretical calculations, the surface plasmonlike resonances on deep and asymmetric gold gratings are reinvestigated and assigned to two classes possessing different characteristic symmetry properties. Reflectivity measurements on deep grating structures with varying depth and asymmetry allow for a detailed study of the influence of these parameters on the lowest-order resonances as well as the experimental observation of a higher-order resonance.
\end{abstract}

DOI: 10.1103/PhysRevB.65.125415

PACS number(s): 73.20.Mf, 78.66.-w, 42.25.Fx, 42.70.Qs

\section{INTRODUCTION}

Metal surfaces that are periodically corrugated on a length scale of the order of the wavelength of light show a very peculiar response to incident light. Sharp minima in the intensity of the light reflected from such a surface are observed when either the wavelength or the experimental geometry is continuously varied. The excitation of surface plasmon resonances, electromagnetic surface waves on the interface between a metal and a dielectric, is one important reason for the richness of the optical response of metal gratings. On shallow gratings, the observed minima can be explained to a good degree of accuracy by the assumption that the surface plasmon resonance has a fixed dispersion, which is not influenced by the grating. ${ }^{1}$ The main effect of the grating is the introduction of higher diffracted orders, which may serve as a channel to transfer energy between the surface plasmon at the interface and the plane light waves in free space. Coupling is established whenever the in-plane momentum of the diffracted order matches the momentum of the surface plasmon. As a consequence, sharp minima are observed in the reflected intensity whenever the experimental geometry fulfills the matching condition. It is well known, ${ }^{2-8}$ though, that the effect of the grating on the surface plasmon resonance cannot be neglected when the experimental geometry is such that the surface plasmons would be excited simultaneously in two diffracted orders. In this case, the plasmon-plasmon interaction mediated by the grating leads to the formation of electromagnetic surface resonances that differ from the "ordinary" surface plasmon both in their in-plane momentum and in their width. This effect is called the "photonic band gap" by analogy with the band gaps in the dispersion of phononic and electronic states in the three-dimensional lattice of a solid. Recently, numerical tools have become available that allow the theoretical investigation of metal gratings with both high modulation and high asymmetry, ${ }^{9}$ based on the Chandezon et al. ${ }^{10}$ approach. The impact of an increased grating amplitude on the optical response of the grating has been theoretically modeled by various authors. ${ }^{11-16}$ In addition to the enhanced plasmon-plasmon interaction on deeper gratings that can be expected intuitively, new types of surface plasmon-related resonances have been predicted on deep grating structures. The main focus of this paper lies with an experimental investigation of these effects on deep grating structures of different degrees of asymmetry. Before discussing the experimental data in Sec. IV, though, the problem is treated theoretically based on numerical calculations in Sec. II. A reinterpretation of the photonic band gap on shallow diffraction gratings focuses on the symmetry properties of the electromagnetic problem and serves as a basis for a better understanding of the calculated response of deep structures. In this framework, some of the features that are found in the subsequent investigation of deep gratings can be assigned to effects that are known from literature.

\section{THEORETICAL INVESTIGATIONS}

\section{A. Grating structure and reflection geometry}

The sample under investigation is sketched in Fig. 1. The gold and the ambient (air) are described by a homogenous dielectric function, which is taken to be 1 for air. The optical response of the gold is described by a dielectric function $\epsilon(\omega)$ with a Drude-type behavior ${ }^{17}$ with a plasma frequency of $\omega_{p}=1.2 \times 10^{16} \mathrm{~s}^{-1}$ and a collision time of $\tau=7$ $\times 10^{-15}$ s. $\omega$ is the frequency of the light.

$$
\epsilon(\omega)=1-\frac{\omega_{p}^{2}}{\omega^{2}+i \frac{\omega}{\tau}}
$$

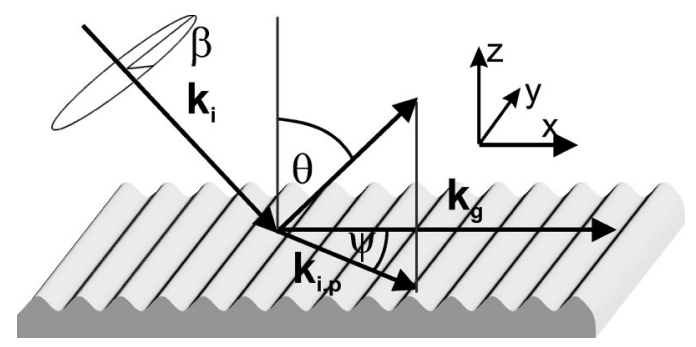

FIG. 1. Sketch of the experimental geometry in a reflection experiment on a grating. The direction of the incident plane wave is defined by the polar angle $\theta$ and the azimuthal angle $\psi$. The wave vector of the incident beam, $\mathbf{k}_{i}$, its projection on the surface, $\mathbf{k}_{i, p}$, and the reciprocal grating vector, $\mathbf{k}_{g}$, are indicated as well as the choice of the Cartesian coordinate system. The angle $\beta$ describes the linear polarization of the incident beam. 
with the plasma frequency $\omega_{p}$, which is given as

$$
\omega_{p}^{2}=\frac{4 \pi n_{e} e^{2}}{m_{e}}
$$

with the charge $e$ and the mass $m_{e}$ of an electron and the electron density $n_{e}$. The parameters $\omega_{p}$ and $\tau$ are chosen to give an optimum description of the literature ${ }^{18}$ values in the investigated spectral region. In a reflection experiment, the grating is illuminated by a plane electromagnetic wave, characterized by its wave vector $\mathbf{k}_{i}$. The polar angle $\theta$, the azimuthal angle $\psi$, and the wavelength $\lambda$ of the incident light can be varied as well as its linear polarization, which is described by the angle $\beta$.

The calculations that are presented below all refer to light that falls on the grating structure along the surface normal $\left(\theta=0^{\circ}\right)$. The electrical-field vector is perpendicular to the grating grooves $\left(\psi=0^{\circ}, \beta=0^{\circ}\right)$.

The interface between the two media is described by a Fourier series with amplitudes $A_{m}$ and phases $\phi_{m}$ in an oblique coordinate frame (compare Ref. 9)

$$
z_{o b}\left(x_{o b}\right)=\sum_{m=1}^{\infty} A_{m} \sin \left(m k_{g} x_{o b}+\phi_{m}\right),
$$

where $z_{o b}$ and $x_{o b}$ are the coordinates in the oblique frame and $k_{g}$ is the modulus of the reciprocal grating vector which equals $2 \pi / \Lambda$ where $\Lambda$ is the grating pitch. The Cartesian representation of the interface is obtained by applying the transformation

$$
\begin{gathered}
z=z_{o b} \sin (\alpha), \\
x=x_{o b}+z_{o b} \cos (\alpha)
\end{gathered}
$$

with the obliquity angle $\alpha$. All calculations presented here are based on the algorithm that was developed by Preist et al. ${ }^{9}$ which allows for the calculation of the reflectivity and the electromagnetic near field of a grating structure that is specified by the parameters that have been introduced in this section.

\section{B. Shallow gratings}

The resonances at the photonic band gap on shallow gratings at normal incidence have been intensely studied as was pointed out in the Introduction. The case of a shallow grating is considered here again in some detail because the symmetry properties of the magnetic-field distribution are an important prerequisite for the understanding of the resonances on deep grating structures that will be investigated later.

In Fig. 2, calculated reflectivities for different grating shapes are shown. The profiles are composed according to Eq. (2.3) of a maximum of two nonvanishing Fourier components. By choosing different phases $\phi_{2}$ of the second harmonic component, gratings with mirror symmetry with either broad troughs (c) or broad peaks (d) can be constructed as well as structures without mirror symmetry (b).

In the absence of a second harmonic component ( $\mathrm{a}, A_{2}$ $=0 \mathrm{~nm})$, the resonance wavelength of the surface plasmon is exactly where it is expected from the momentum-matching

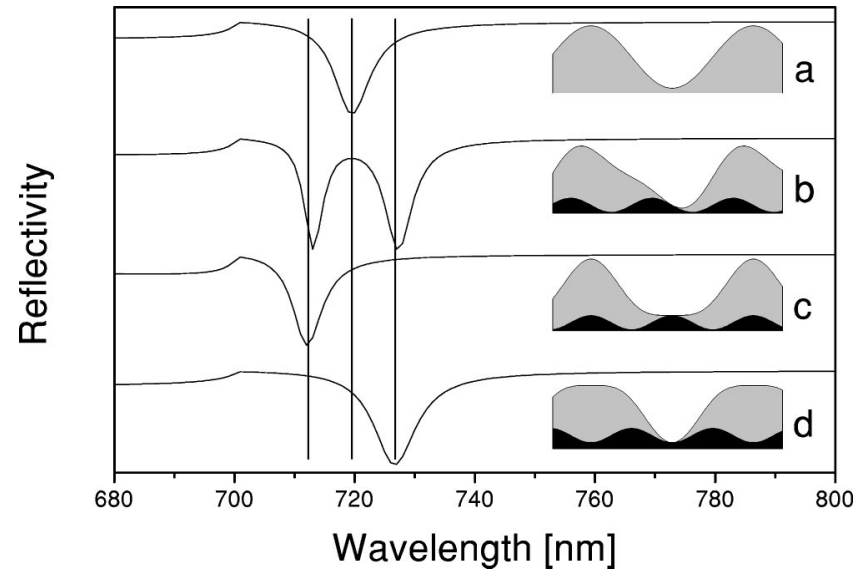

FIG. 2. Wavelength-dependent reflectivity at normal incidence ( $\theta=0^{\circ}$ ) of the grating structure with $\Lambda=700 \mathrm{~nm}, A_{1}=20 \mathrm{~nm}$ and (a) $A_{2}=0 \mathrm{~nm}$, (b) $A_{2}=5 \mathrm{~nm}$ and $\phi_{2}=0^{\circ}$, (c) $A_{2}=5 \mathrm{~nm}$ and $\phi_{2}=270^{\circ}$, (d) $A_{2}=5 \mathrm{~nm}$ and $\phi_{2}=90^{\circ}$. The vertical lines allow for better comparison of the minima for the different cases. The grating profiles are presented graphically together with the second harmonic component. The depth-to-pitch ratio is strongly exaggerated.

approach $(\lambda=720 \mathrm{~nm})$. For the asymmetric grating $\left(\mathrm{b}, A_{2}\right.$ $=5 \mathrm{~nm}, \phi_{2}=0^{\circ}$ ), two resonances are clearly seen, one is shifted to smaller wavelengths relative to the original resonance and has become narrower, the other one has shifted to longer wavelengths and has become broader. This splitting is the photonic band gap of surface plasmons. This example illustrates the importance of the second harmonic component being responsible for the plasmon-plasmon coupling, which is a prerequisite for the existence of a photonic band gap.

Sarid ${ }^{19}$ observed a similar phenomenon on a thin metal film. There, the interaction of the two surface plasmons on the two sides of the film led to the formation of two coupled resonances of different widths. In his paper, these resonances were labeled as the long-range surface plasmon and the short-range surface plasmon according to their propagation length. Although the mechanism of plasmon-plasmon coupling is different in our case, the resulting resonances exhibit different widths as well. For this reason, the terms grating coupled long-range/short-range surface plasmon (GLRP/ GSRP) have been used to label the coupled resonances investigated in our work.

The two symmetric gratings support only one resonance each. The resonance wavelength on the broad-peak profile $\left(A_{2}=5 \mathrm{~nm}, \phi_{2}=90^{\circ}\right)$ is identical to that of the GSRP whereas the resonance wavelength on the broad-trough profile $\left(A_{2}=5 \mathrm{~nm}, \phi_{2}=270^{\circ}\right)$ is that of the GLRP.

For a deeper understanding of the nature of these surface resonances, it is helpful to analyze their electromagneticfield distribution. The symmetry of the problem leads to a magnetic field, which has only one nonvanishing component $H_{y}$. For this reason, in the following discussion the resonances are described in terms of their magnetic-field distribution for the sake of simplicity.

Figure 3 shows the magnetic-field distributions for coupled resonances that are observed on (a) a purely sinusoidal grating, (d) a broad-valley grating, and (e) a broad- 

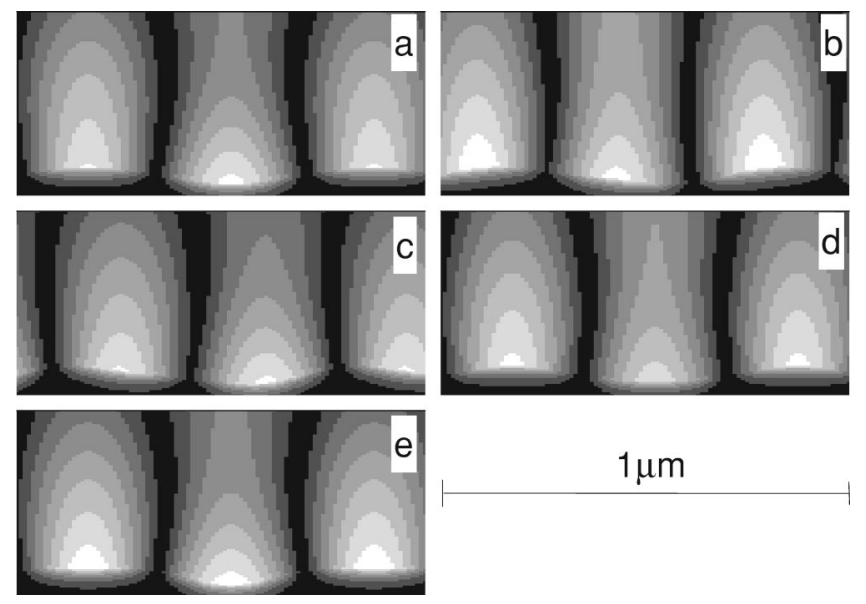

$1 \mu \mathrm{m}$

FIG. 3. Modulus of the magnetic field upon excitation of surface resonances at $\theta=0^{\circ}$ for a grating with $\Lambda=700 \mathrm{~nm}$; amplitude of the first harmonic $A_{1}=20 \mathrm{~nm}$, and amplitude of the second harmonic are as specified: (a) $A_{2}=0 \mathrm{~nm}, \lambda=720 \mathrm{~nm}$ (normal surface plasmon), (b) $A_{2}=5 \mathrm{~nm}, \phi_{2}=0^{\circ}, \lambda=712 \mathrm{~nm}$ (GLRP), (c) $A_{2}$ $=5 \mathrm{~nm}, \phi_{2}=0^{\circ}, \lambda=727 \mathrm{~nm}$ (GSRP), (d) $A_{2}=5 \mathrm{~nm}, \phi_{2}=90^{\circ}$, $\lambda=727 \mathrm{~nm}$ (GSRP), (e) $A_{2}=5 \mathrm{~nm}, \phi_{2}=270^{\circ}, \lambda=712 \mathrm{~nm}$ (GLRP). The grating shape can be roughly seen from the black profile of vanishing field at the bottom of each figure.

peak grating. For the blazed grating, field distributions corresponding to both resonances supported by this structure are shown $(\mathrm{b}, \mathrm{c})$. For geometrical reasons, only the $y$ component of the magnetic field (the one parallel to the grating grooves) does not vanish, therefore the magnetic field can be represented as a (complex) scalar function. In all field distributions, the modulus of this function is plotted.

Strong modulation of the magnetic field along the interface is observed with two intensity maxima per fundamental oscillation of the function describing the interface. The positions of these maxima depend on the surface profile. For the pure-sine case (a), the broad-peak case (d), and the broadvalley case (e) they are located in the valleys and on the peaks of the fundamental component. Nodes are observed in the slopes of the surface profile. The two resonances on the blazed gratings have similar field distributions but the field maxima are shifted by roughly (1/8)th of a period with respect to the fundamental. The GLRP is characterized by a shift to the left (b) whereas the GSRP is shifted to the right (c).

Barnes et al. ${ }^{3}$ pointed out that the relative position of electrical-field maximum and second harmonic component of the grating profile determines the direction of the shift of the coupled resonance relative to the unperturbed position. Obviously, the situation can be equally well described in terms of the magnetic-field: The GSRP has its magnetic-field maxima in the troughs of the second harmonic component whereas the GLRP's magnetic field is concentrated on the peaks of the second harmonic.

The lack of one coupled resonance on the symmetric grating profiles can be explained in terms of the symmetry properties of the electromagnetic boundary problem. These resonances would have nodes of the magnetic-field intensity on the peaks and in the troughs of the fundamental component in order to have the correct position relative to the second harmonic of the grating profile. Such a field distribution would have odd symmetry with respect to the symmetry plane of the grating profile because the complex number $H_{y}$ (the only nonvanishing component of $\mathbf{H}$ ) is phase shifted by $180^{\circ}$ upon each crossing of a nodal plane. (A consequence of the continuity of the first derivative of the magnetic field.) The electromagnetic boundary problem has even symmetry with respect to these planes when described in terms of the magnetic field vector, which is lying parallel to the grating grooves and does not change its sign upon reflection at the mirror plane. As a consequence, a solution corresponding to the "forbidden" resonances can be excluded by this symmetry consideration. As soon as the grating loses mirror symmetry, there are no restrictions to the symmetry of the electromagnetic-field distributions. That is why both resonances are coupled to incident light on a blazed grating.

It should be noted that the same analysis could be performed on the basis of the electrical field by considering separately the two nonvanishing components in the $\mathbf{x}$ direction (odd symmetry) and $\mathbf{z}$ direction (even symmetry). Both the electrical- and the magnetic-field distribution determine the solution of the electromagnetic problem completely, the choice of $\mathbf{H}$ in this paper is just for simplicity.

\section{Deep gratings with mirror symmetry}

Now, after having discussed some features of coupled resonances in the relatively simple case of a shallow grating, the focus is on the additional effects that come into play when the grating amplitude is increased. It is most instructive to vary both the wavelength of the incident light and the grating amplitude continuously and investigate the dispersion of the resonances, which can be observed as areas of reduced reflectivity in the plane that is spanned by these two parameters. The grating amplitude is varied by applying a linear scaling factor to all Fourier components $A_{i}$ of one grating profile in Eq. (2.3), thus keeping the ratios between the individual amplitudes fixed. The fundamental amplitude $A_{1}$ is used as a measure for the total grating height. In order to describe asymmetric grating profiles, an obliquity angle $\alpha \neq 90^{\circ}$ is used [compare Eq. (2.4)].

In Fig. 4, the reflectivity of a deep broad-peak grating (the precise grating profile is specified in the figure caption) is plotted as a gray scale as a function of the wavelength of the incident light and the grating amplitude.

The pseudocritical edge at $\lambda=700 \mathrm{~nm}$, where the first diffracted orders become evanescent, is clearly seen as a sharp rise in intensity. For longer wavelengths, the grating is of zero order, which means that all diffracted orders are evanescent. In this region, four extended dark stripes, indicating low reflectivity, are observed. These resonances shift to the infrared for increasing grating amplitudes.

The analysis of the magnetic-field distribution across the grating structure in these resonances is shown in Fig. 5.

The distributions (a)-(c) are taken at about the same wavelength for increasing amplitude of the grating as indicated in Fig. 4. They correspond to the first, second, and fourth branch. It can be seen that each branch has a unique 


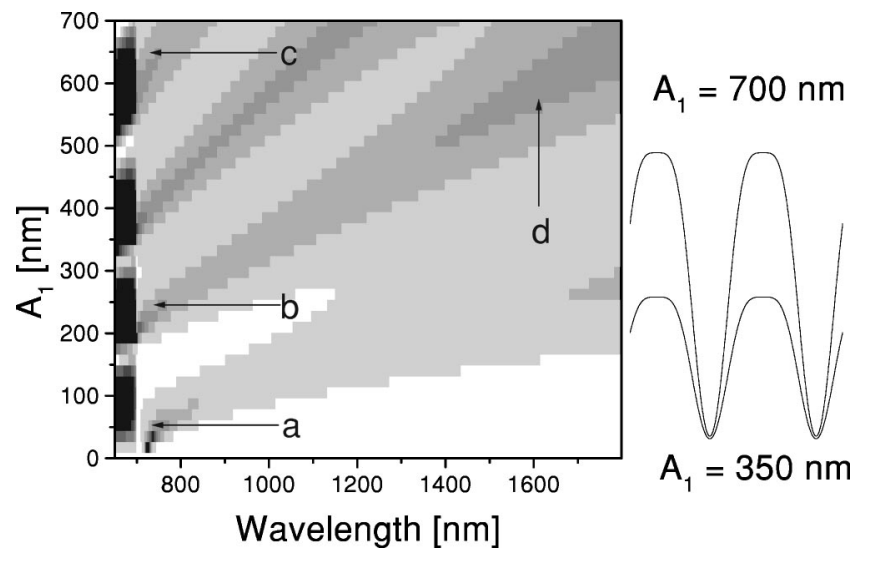

FIG. 4. Calculated reflectivity of a grating $(\Lambda=700 \mathrm{~nm})$ of the form shown on the right as a function of grating amplitude and wavelength of the incident light $\left(\theta=0^{\circ}\right)$. The gray values indicate different reflectivities from 0.5 (black) to 1 (white). The arrows indicate for which parameters $\left(\lambda, A_{1}\right)$ field distributions are calculated. The maximum grating profile has the Fourier components $A_{1}=700 \mathrm{~nm}, A_{2}=175 \mathrm{~nm}, \phi_{2}=90^{\circ}$. The grating profile has mirror symmetry $\left(\alpha=90^{\circ}\right)$.

topology of the magnetic-field distribution, with a characteristic number of nodal planes and maxima. For each step upwards, one nodal plane, spanning the grating groove, is added. Figure 5(d) shows that within the same branch as (b) the number of maxima and nodes of the magnetic field inside the groove does not change. In the following, these higher resonances will be called $\mathrm{ME}_{i}$ ("magnetically even") where the integer $i$ denotes the number of separated field maxima inside the grating groove.

If we recall the symmetry constraints to an allowed solution of the electromagnetic boundary problem, it is seen that these "ladders" have the required even symmetry. It should be noted that they are not the only possible solutions. LopezRios et al. ${ }^{13}$ have investigated a shallow grating with very widely separated grooves where additional nodes show up on top of the grating peaks.

Predictions for the type of resonances on deep diffraction gratings that are discussed here can be found in the literature

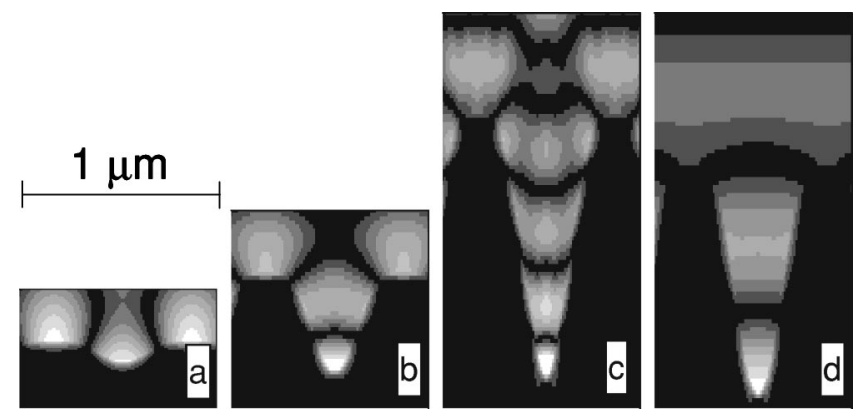

FIG. 5. Calculated spatial distribution of the magnetic field strength as gray values (white indicating high magnetic-field strength) for some selected resonances that are indicated in Fig. 4. The corresponding wavelengths and fundamental amplitudes are (a) $\lambda=750 \mathrm{~nm}, A_{1}=52.2 \mathrm{~nm}$, (b) $\lambda=757 \mathrm{~nm}, A_{1}=245 \mathrm{~nm}$, (c) $\lambda$ $=750 \mathrm{~nm}, A_{1}=648 \mathrm{~nm}$, (d) $\lambda=1595 \mathrm{~nm}, A_{1}=578 \mathrm{~nm}$. as well. The first of these resonances is identical to the GSRP in the limit of very small grating amplitudes. For higher amplitudes, the field becomes more concentrated inside the groove at the expense of the field strength on the peak of the grating. This resonance was described by various authors. ${ }^{11,12}$

The entire family of resonances that is found on deeper gratings was described by Watts et al. ${ }^{20}$ and Sobnack et al., ${ }^{14}$ the latter giving a good intuitive interpretation. The electromagnetic surface modes propagate along the grating tops as surface plasmons while they have to be described as waveguide modes inside the groove. The total phase that is picked up by such a mode between two equivalent points on the grating must be equal to a multiple integer of $2 \pi$. An approximate analytical calculation based on this model leads to a good agreement compared to the rigorous modeling. Maradudin et al. ${ }^{21}$ considered a single groove in an otherwise plane metal surface and found similar resonances.

Currently, there is much interest in the transmission through slits in metal layers, which are of the order of the wavelength of light or smaller. ${ }^{16,22}$ A strong enhancement of the transmission due to the excitation of localized resonances inside the grooves is predicted by numerical calculations, this effect being strongly related to the resonances discussed above.

\section{Deep asymmetric gratings}

From the investigations on shallow gratings it could be already anticipated that a broken symmetry will make the optical response of deep gratings richer. Some theoretical investigations on this problem were performed by Preist et al. ${ }^{9}$ Wanstall et al. ${ }^{15}$ concentrated on highly overhanging gratings and found a family of resonances similar to the ladder of the $\mathrm{ME}_{i}$ resonances that was discussed above. Still, there is no investigation on slightly blazed, deep gratings in the literature. For this reason, the additional features that are introduced by the asymmetry of the grating and their evolution when the asymmetry is increased are discussed in this section.

The asymmetry is introduced to the grating shape by choosing an obliquity angle $\alpha \neq 90^{\circ}$ [of Eq. (2.4)].

Already a very small blaze $\left(\alpha=80^{\circ}\right)$ has a drastic effect on the resonance structure as can be seen in Fig. 6 .

The first two $\mathrm{ME}_{i}$ resonances are visible in this plot only very weakly due to the small deviation of the profile from pure sine. Additionally, a very deep and narrow resonance appears, which was not observed for gratings with mirror symmetry. In the limit of a shallow grating this new feature is identical to the GLRP. A view of the magnetic-field distributions (Fig. 7) reveals why this resonance appears only on blazed gratings. The magnetic-field distributions show almost odd symmetry, which is only possible because the mirror symmetry of the grating is slightly broken.

This finding allows for an extension of the interpretation of the deep grating as an array of small optical waveguides as given by Sobnack et al. ${ }^{14}$ It can be inferred that the waveguides that are discussed there are able to support two families of surface plasmon-related resonances. One family 


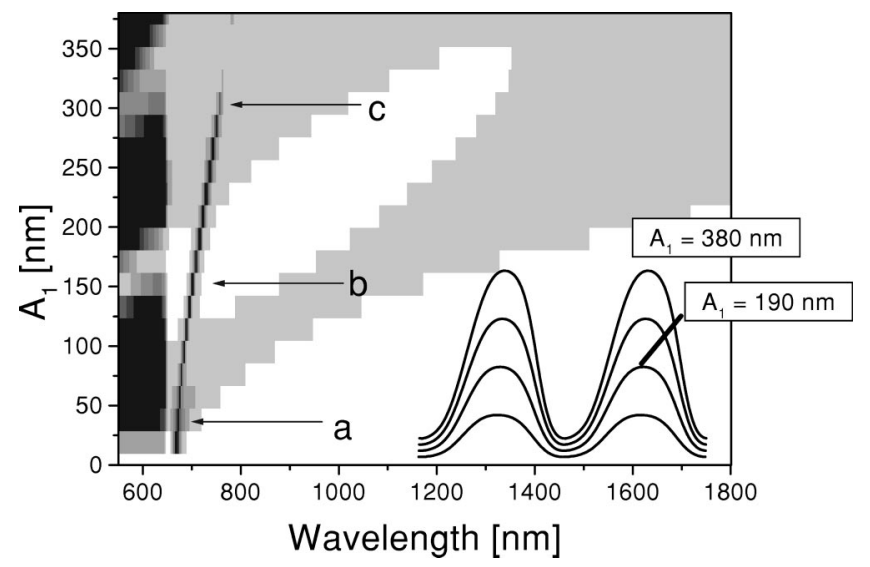

FIG. 6. Calculated reflectivity of a grating $(\Lambda=650.8 \mathrm{~nm})$ of the form shown on the right as a function of the grating amplitude and the wavelength of the incident light $\left(\theta=0^{\circ}\right)$. The gray values indicate different reflectivities from 0.5 (black) to 1 (white). The arrows indicate for which parameters the field distributions are calculated. The maximum grating profile has the Fourier components $A_{1}=380 \mathrm{~nm}, \quad A_{2}=10.6 \mathrm{~nm}, \quad \phi_{2}=26.3^{\circ}, \quad A_{3}=23.4 \mathrm{~nm}, \quad \phi_{3}$ $=36.3^{\circ}$. The obliquity angle is $\alpha=80^{\circ}$.

$\left(\mathrm{ME}_{i}\right)$ is characterized by a magnetic field with even symmetry and an electrical field with odd symmetry, it can be regarded as a standing $\mathrm{TM}_{0}$ waveguide mode. On shallow broad-peak gratings, this family is represented by the GSRP. The other family can be regarded as $\mathrm{TM}_{1}$ modes inside the grooves. It is characterized by a magnetic field with odd symmetry and an electric field with even symmetry. It is not coupled to free electromagnetic radiation incident normally on a mirror-symmetric grating. Breaking of the symmetry of the grating profile allows coupling and, as a consequence, its observation. It should be noted that due to the slightly broken symmetry of the grating profile, the even or odd symmetry of these modes is not exact. But if the grating asymmetry is not too large, these assignments are still helpful to understand the observed resonance pattern.

An alternative way to excite modes with a magnetic-field distribution with odd symmetry is described by Watts et al. ${ }^{20}$ They report the observation of a resonance that is coupled with TM light in a geometry where the plane of incidence is parallel to the grating grooves $\left(\psi=90^{\circ}\right)$ and the polar angle $\theta$ is different from $0^{\circ}$. There, the additional existence of higher orders of this family of resonances for very deep gratings is shown in the calculations. This experimental geometry preserves the symmetry of the grating but offers an ex-
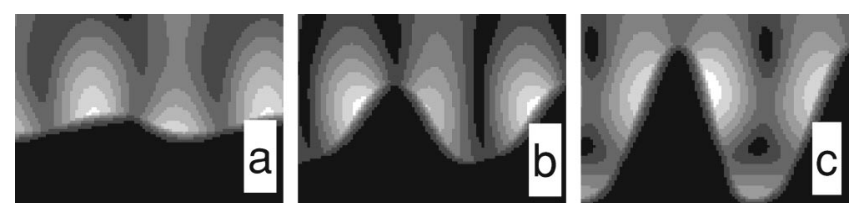

FIG. 7. Spatial distribution of the magnetic-field strength as gray values (white indicating high magnetic-field strength) for some selected resonances as indicated in Fig. 6), (a) $\lambda=673 \mathrm{~nm}, A_{1}$ $=38 \mathrm{~nm}$, (b) $\lambda=703 \mathrm{~nm}, A_{1}=152 \mathrm{~nm}$, (c) $\lambda=757 \mathrm{~nm}, A_{1}$ $=304 \mathrm{~nm}$. Each section is $1 \mu \mathrm{m}$ wide.

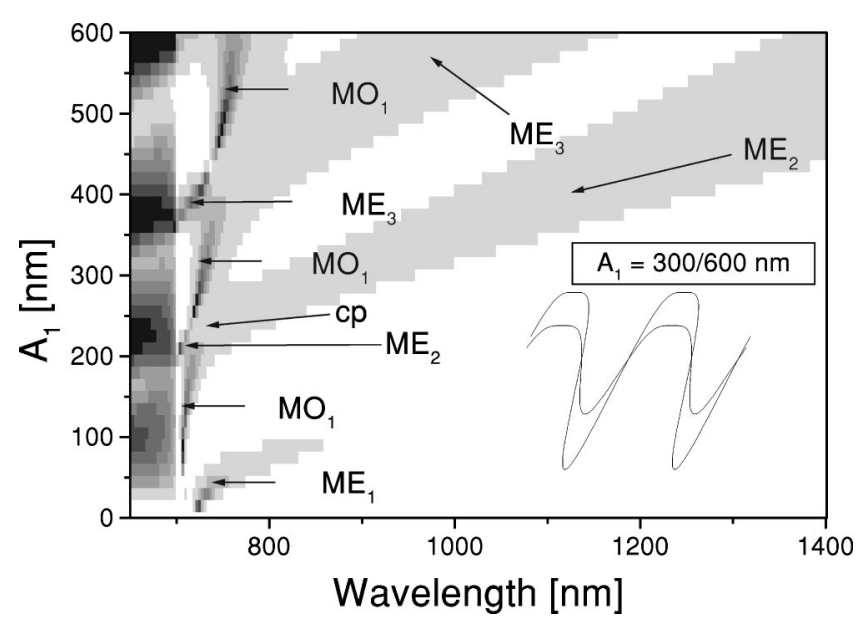

FIG. 8. Calculated reflectivity of a grating $(\Lambda=650.8 \mathrm{~nm})$ of the form as shown on the right as a function of grating amplitude and wavelength of the incident light $\left(\theta=0^{\circ}\right)$. The gray scale indicates different reflectivities from 0 (black) to 1 (white). The arrows indicate for which parameters the field distributions are calculated. The maximum grating profile has the Fourier components $A_{1}$ $=600 \mathrm{~nm}, A_{2}=150 \mathrm{~nm}, \phi_{2}=90^{\circ}$. The obliquity angle is $\alpha$ $=70^{\circ}$. The assignments on the arrows are explained in the text.

citing magnetic field with odd symmetry. In the further investigations this type of resonance will be called "magnetically odd" $\mathrm{MO}_{i}$.

\section{E. Interaction between the two families of resonances}

When the appropriate grating profile is chosen, the two families of resonances with even and odd symmetry may appear with significant strength simultaneously. This makes the reflectivity map of such a grating relatively complex. Figure 8 shows calculated reflectivities for a broad-peak profile, which is tilted by an obliquity angle of $\alpha=70^{\circ}$. The first three resonances with a magnetic field with even symmetry $\left(\mathrm{ME}_{1}, \mathrm{ME}_{2}, \mathrm{ME}_{3}\right)$ are seen as broad areas of slightly reduced reflectivity which shift quickly to the infrared for increasing grating amplitude. Close to the pseudocritical edge, a rich structure is seen that can be interpreted as follows: The areas of low reflectivity, marked with a $\mathrm{MO}_{1}$ correspond to the first resonance with a magnetic field of odd symmetry. The minima right at the pseudocritical edge belong to resonances with even-symmetric magnetic field again. That means: a crossing of the $\mathrm{MO}_{1}$ resonance with the $\mathrm{ME}_{2}$ and $\mathrm{ME}_{3}$ resonances leads to this reflectivity behavior.

Of course, it must be checked whether the assignment given above reflects the real physics. The magnetic-field distributions of the $\mathrm{MO}_{1}$ resonance and the $\mathrm{ME}_{2}$ resonance are shown in Fig. 9 right before and after crossing each other. The "crossing point" is indicated in Fig. 8 as cp.

It can be seen that the symmetry of the field distributions is highly distorted. Nonetheless, the topology of the nodal planes still reflects the original symmetry: the plots (a) and (d) correspond to the $\mathrm{ME}_{2}$ resonance to the left and right of the crossing point that is indicated in Fig. 8. The characteristic nodal line across the grating groove is visible in both cases. In contrast, (b) and (c), which correspond to the $\mathrm{MO}_{1}$ 

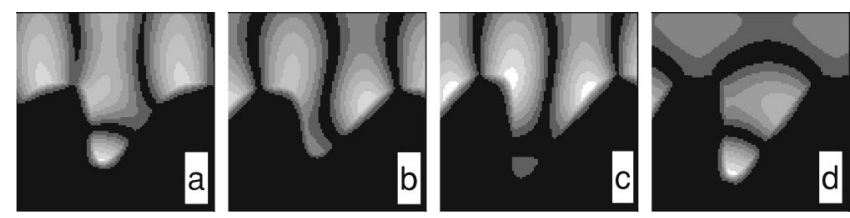

FIG. 9. Spatial distribution of the magnetic-field strength as gray scales (white indicating high magnetic-field strength) for some selected resonances as indicated in Fig. 8), (a) $\lambda=704 \mathrm{~nm}, A_{1}$ $=180 \mathrm{~nm}$, (b) $\lambda=717 \mathrm{~nm}, A_{1}=210 \mathrm{~nm},(\mathrm{c}) \lambda=721 \mathrm{~nm}, A_{1}$ $=270 \mathrm{~nm}$, (d) $\lambda=854 \mathrm{~nm}, A_{1}=270 \mathrm{~nm}$. Each section of the grating is $1 \mu \mathrm{m}$ wide.

resonance below and above the crossing point, clearly show a nodal line that is lying on the "symmetry axis" of the grating, which is typical for odd symmetry. One further observation is made in the field distribution (c): on the extreme bottom of the profile, a weak portion of magnetic field has built up with even symmetry. Upon each subsequent crossing, a new horizontal nodal plane will be added, which leads to the formation of resonances that may be described as even type at the bottom and odd type near the peaks of the profile.

Investigations on a strongly overhanging grating are reported by Wanstall et al. ${ }^{15}$ In this case, the approximate assumption of a symmetry of the grating profile and therefore the classification of the resonances as is presented above, fails.

\section{F. Summary of the theoretical investigations}

The resonances that lead to absorption of light falling vertically on metallic gratings were investigated theoretically for a Drude-type metal (gold). It was found that, on symmetric gratings, two classes of resonances may exist with magnetic-field distributions that exhibit either even or odd symmetry. Only the even ones couple to light at normal incidence. By introduction of a slight asymmetry to the grating profile, the resonances can still be roughly classified like in symmetric gratings. Now, both types are observed in the same experimental geometry and mutual interactions take place.

\section{PREPARATION OF DEEP GRATING STRUCTURES}

\section{A. Preparation}

In order to obtain gratings that are as deep as possible, the preparation routine was chosen that is schematically depicted in Fig. 10.

Standard microscope slides (obtained from Menzel) are carefully cleaned and a thin chromium layer followed by a $\approx 1000$-nm-thick gold layer and another chromium layer of thickness $200 \mathrm{~nm}$ are deposited on the substrate by thermal evaporation. The first chromium layer enhances the adhesion of the gold to the surface, the second is chosen as masking material because it is very resistive to ion-etching processes, the etching rate in gold is roughly four to five times higher than in chromium. For the fabrication of highly asymmetric gratings, a large contrast in the etching resistance between the mask and the material into which the structure is finally written is needed. The thickness of the gold layers is required

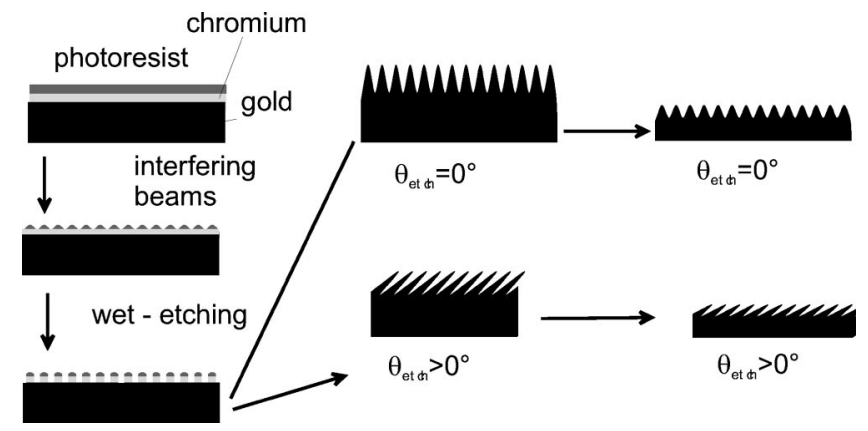

FIG. 10. Sketch of the different steps for the preparation of the deep gold gratings.

in order to simplify the evaluation: the gold should be thick enough to allow for the assumption of an infinite thickness for the comparison to theory. The evaporation materials were obtained from Balzers with a purity of $99.99 \%(\mathrm{Au})$ and 99.9\% ( Cr).

This system serves as a support for a photoresistant film (Shipley Microposit) of $100 \mathrm{~nm}$ thickness which is deposited by spin coating. A holographic grating is written into the photoresist film. The development is long enough to ensure that the photoresistant structure has evolved from a coherent layer to separated stripes. Subsequently, the sample is exposed to an aqueous solution of ammonium cerium (IV) nitrate (Aldrich, 99\%, $12 \mathrm{~g}$ in $50 \mathrm{ml}$ water) for $70 \mathrm{sec}$. This step serves to transfer the photoresistant structure into the chromium. As a next step, the sample is treated by reactive ion-beam etching until the chromium is completely removed. By choosing the etching angle $\theta_{\text {etch }}$ between the direction of the ion beam and the surface normal of the grating to be between $0^{\circ}$ (normal incidence) and $40^{\circ}$, gratings with different degrees of asymmetry can be obtained. The orientation of the grating is chosen so that the ion beam is orthogonal to the grating grooves. To some extent, the grating amplitude can now be decreased by etching further into the pure gold grating.

\section{B. Characterization}

The gratings that were produced this way were characterized with a scanning electron microscope (Leo electron microscopy, model 912) to obtain information about the grating profile completely independent from the optical measurements. The samples were broken along a line orthogonal to the grating grooves from the backside of the substrate. Figure 11 shows the grating profile for different etching angles. It is clearly seen that with this technique, it is possible to obtain gold gratings with a depth-to-pitch ratio close to 1 . The grating shape can be varied from a mirror-symmetric profile when etched under $\theta_{\text {etch }}=0^{\circ}$ to overhanging structures when etched under $\theta_{\text {etch }}=40^{\circ}$. From images like these, the grating shape had to be extracted. This was done by drawing a curve by hand into the images that reproduced the surface profile as good as possible. This curve was then transformed into discrete points $z(x)$. A least-squares fit to the average of five data sets that were obtained on different grating peaks gave finally the parameters for the theoretical 


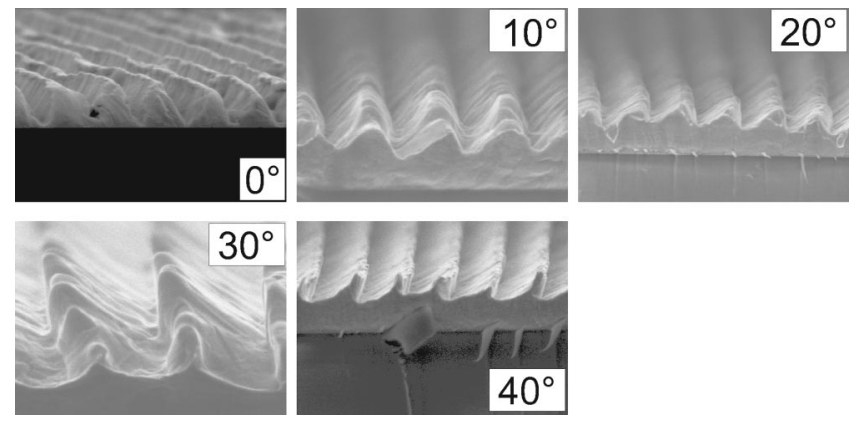

FIG. 11. Scanning-electron-images of grating profiles obtained under the etching angles $\theta_{\text {etch }}$ as indicated in the graph.

description of the profile. The first three amplitudes $A_{i}$ and phases $\phi_{i}$ of the Fourier sum [Eq. (2.3)] and the obliquity angle $\alpha$ were obtained that way. One major disadvantage of this characterization technique should be mentioned: the sample must be destroyed to allow for the measurements. Therefore, it was not possible to characterize the identical sample for different exposure times in the reactive ion-beam etcher.

\section{EXPERIMENTAL RESULTS}

In this section, reflectivity measurements on gratings with varying blaze and amplitude are discussed and compared to the theoretical predictions. The depth and degree of asymmetry was tuned by varying the geometry and exposure time in the etching process as described in Sec. III. The presentation of the results is organized as follows: First, wavelengthdependent reflectivity data are presented that were taken at (almost) normal incidence on asymmetric gratings with the setup that is described in Sec. IV A. For three different degrees of asymmetry, the reflectivities are compared to the theoretical predictions. Additionally, measurements are shown for samples with varying amplitudes for these blazed gratings. In the Sec. IV C, the theoretical prediction of stationarity of the redshifted $\mathrm{ME}_{i}$ resonances is proved experimentally. Section IV D deals with measurements on a symmetric grating where the experimental geometry is varied. And finally, in Sec. IV E an experimental observation that cannot be described with the idealized model of a perfect grating is presented and an explanation is proposed.

\section{A. Optical setup for reflectivity measurements}

The reflectivity of the gratings was measured for linearly polarized light as a function of the wavelength $\lambda$, the polarization $\beta$, and the angle of incidence $(\psi, \theta)$ of the incoming light (compare Fig. 1). In Fig. 12 the optical experimental setup is shown schematically. The light of a xenon light bulb (Osram Xenophot, $300 \mathrm{~W}$ in a Müller SVX 1530 housing) is collimated by a lens onto the slit of a grating monochromator (Chromex $250 \mathrm{SM}$ ). After its exit slit, a lens turns the light into a parallel beam. Linear polarization is fixed by a GlanThompson polarizer (OWIS) and the polarization plane can be deliberately rotated with a Fresnel rhomb (Bernhard

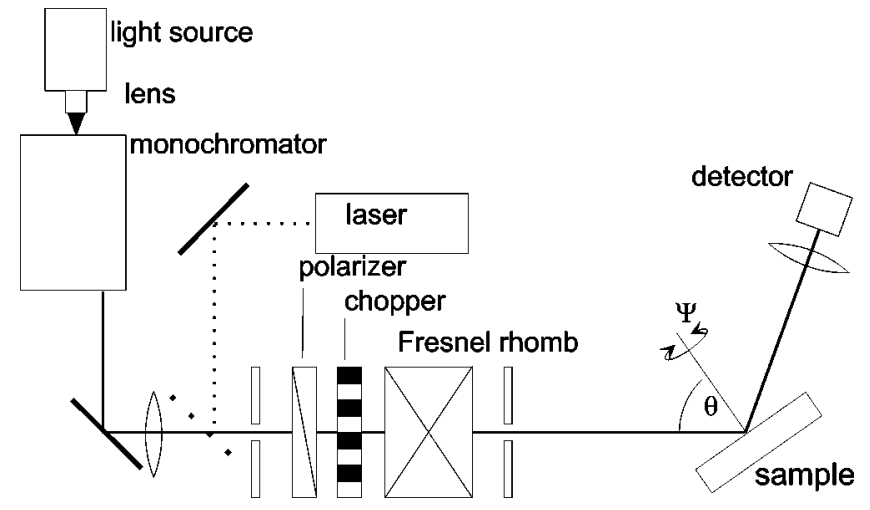

FIG. 12. Sketch of the optical setup for reflectivity measurements.

Halle). Two pinholes select the components of the beam, which are close to the optical axis and are therefore almost parallel.

An appropriate combination of goniometers allows the adjustment of the sample relative to the incident beam, described by the angles $\theta, \psi$ as well as the positioning of the detector to collect the specularly reflected light.

A lens is used to focus the light onto the detector, which is either a silicon photodiode (for $\lambda=500$ to $1100 \mathrm{~nm}$ ) or a germanium photodiode (for $\lambda=1000$ to $1800 \mathrm{~nm}$ ). The lock-in detection technique is used; for this reason the incoming beam is passing an optical chopper.

The spectral intensity for the two fundamental polarizations of the incoming beam is recorded by removing the sample and collecting the light beam that has undergone no reflection. In order to measure reflectivities of the sample, the readings of the lock-in technique in a reflection experiment are normalized to this reference.

\section{B. Blazed gratings at "normal" incidence}

First, the optical response of a grating etched under $\theta_{\text {etch }}=10^{\circ}$, thus possessing a slight asymmetry, is investigated. After the recording of the reflectivity data, the sample was characterized by electron microscopy and an approximate surface profile was determined. The experimental geometry $\left(\theta=4^{\circ}, \psi=90^{\circ}\right)$ is chosen to have the same symmetry as normal incidence. Real normal incidence could not be established for experimental reasons. Numerical calculations show that the change in reflectivity compared to normal incidence is negligible. In the geometry chosen here, it is the TE polarization that has an electrical field perpendicular to the grating grooves and plays therefore the same role as TM polarization in the classical mount $\left(\psi=0^{\circ}\right)$.

Theoretical modeling was done on the basis of grating profiles as determined from the electron-microscopy images. For these model calculations, the dielectric function of gold was modeled as a Drude metal with the parameters given in Sec. II A.

The wavelength-dependent reflectivity is shown in Fig. 13 for illumination with TE and TM polarized light, respectively. For $\lambda$ between 1000 and $1100 \mathrm{~nm}$, the data sets taken with the two detectors overlap. The slight deviation between 


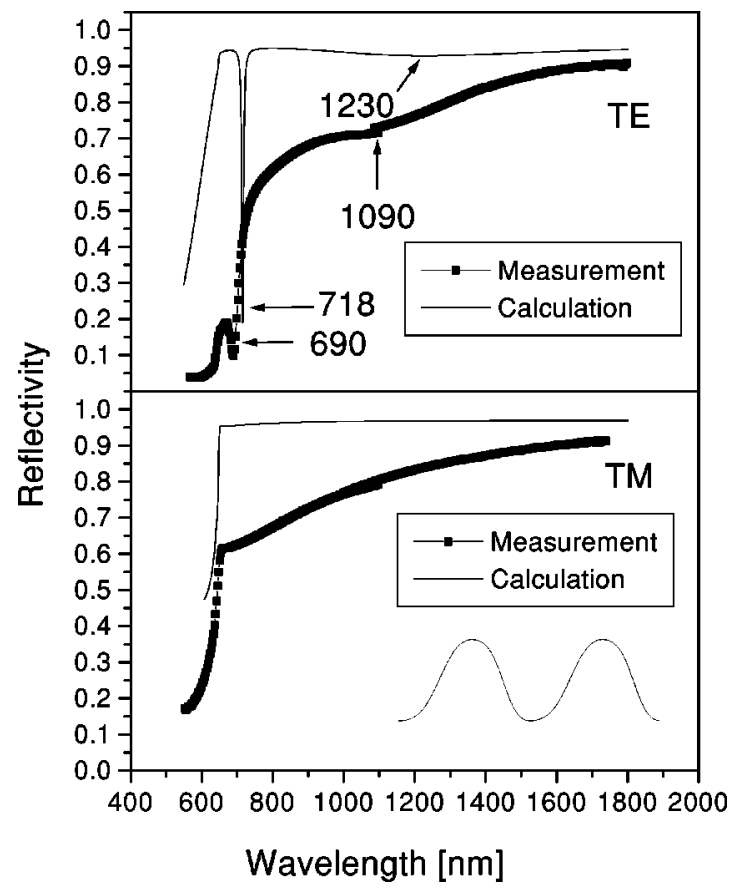

FIG. 13. Wavelength-dependent reflectivity $\left(\theta=4^{\circ}, \psi=90^{\circ}\right)$ from a grating etched under $\theta_{\text {etch }}=10^{\circ}$ for TM and TE polarization. The theoretical curve is based on the grating profile that is shown in the inset.

the two datasets is due to the fact that both detectors have a low efficiency in this wavelength regime, which enhances errors due to stray light. It is clearly seen that although there is quantitative agreement between measurement and modeling, there are some discrepancies with respect to the observed resonances.

The pseudocritical edge observed at $\lambda=650 \mathrm{~nm}$ gives good agreement between theory and experiment as expected. For short wavelengths, the measured reflectivity is much too low, while further in the infrared, the measured values are approaching the theoretical prediction for both polarizations. One possible explanation for this experimental finding is the random roughness introduced by the preparation routine in addition to the geometrically perfect grating structure. This additional roughness is visible in the scanning electron microscopy (SEM) images (see Fig. 11). It results in random scattering of light with wavelengths comparable or smaller than the typical size of the random surface structure, leading to a reduction of the light intensity that is detected. This qualitatively corresponds to the measurement: For increasing wavelength, the effect of surface scattering decreases, which leads to a higher observed intensity. Some more evidence for the importance of scattering on random roughness is found in Sec. IV E.

For illumination with TE light, two resonances can be observed. A very narrow and deep minimum appears around $\lambda=700 \mathrm{~nm}$ which is predicted slightly too far in the infrared. This feature is assigned to the lowest resonance with magnetic field with odd symmetry $\left(\mathrm{MO}_{1}\right)$. The measured minimum appears broader and shallower than in the model calculation, which is due to the finite-wavelength resolution of the setup and the inhomogenity of the real grating.

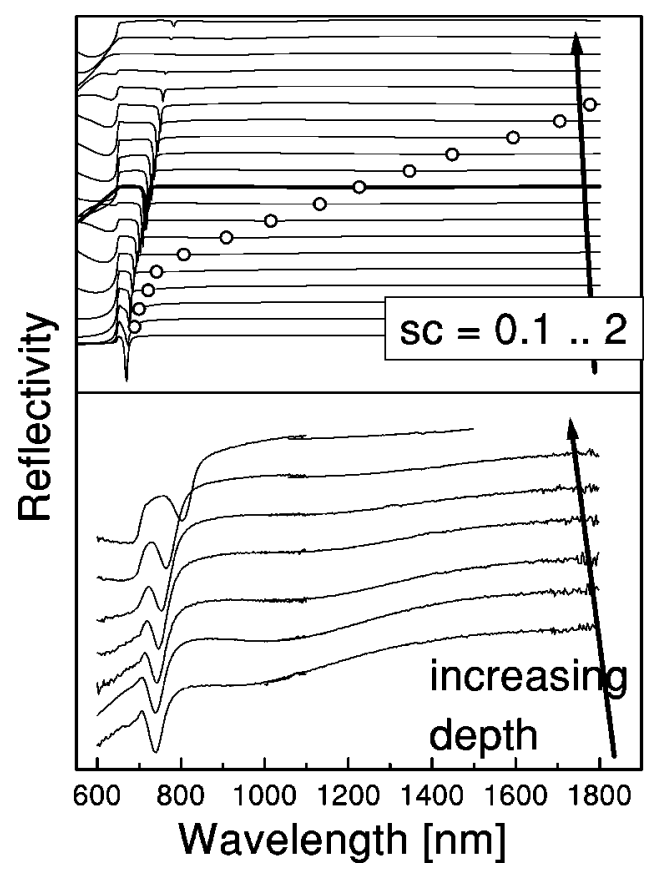

FIG. 14. Modeled (top) and measured (bottom) wavelengthdependent reflectivity $\left(\theta=4^{\circ}, \psi=90^{\circ}\right.$ ) from a grating etched under $\theta_{\text {etch }}=10^{\circ}$ with scaling factors applied to the grating profile underlying the calculation, which vary between 0.1 and 2 . The position of the $\mathrm{ME}_{1}$ resonance is indicated by open circles. The thicker line corresponds to the grating shape as determined by electron microscopy.

The other resonance is much broader and further in the infrared. Again, there is a discrepancy between theory and experiment with respect to its position. Additionally, this resonance appears deeper in the measurement than in the calculation. It is assigned to the strongly redshifted $\mathrm{ME}_{1}$ resonance.

The measurement with TM polarization does not show any resonance minimum, which is in agreement with theory.

The measured dispersion of these two resonances as a function of the grating amplitude is presented in Fig. 14 together with the corresponding calculations. The data were obtained after subsequent etching steps of the same grating. Since it is impossible to obtain REM images without destroying the sample, it was not possible to measure the profiles corresponding to each wavelength scan. The theoretical calculation is based on the profile as obtained from an identically prepared reference sample. In order to account for the decreasing amplitude due to the additional etching steps, a scaling factor was applied to the Fourier sum describing the grating profile [Eq. (2.3)]. Since it is neither known how much the grating amplitude is decreasing by etching into the pure gold, nor if the originally determined grating profile is still applicable for strongly etched gratings, this theoretical treatment cannot give a full quantitative description of the measured data. Nevertheless, it is possible to compare the impact of an increased grating amplitude on the depth and position of the observed resonances.

By comparison of the positions of the two resonances that are visible both in theory and experiment, the calculated re- 


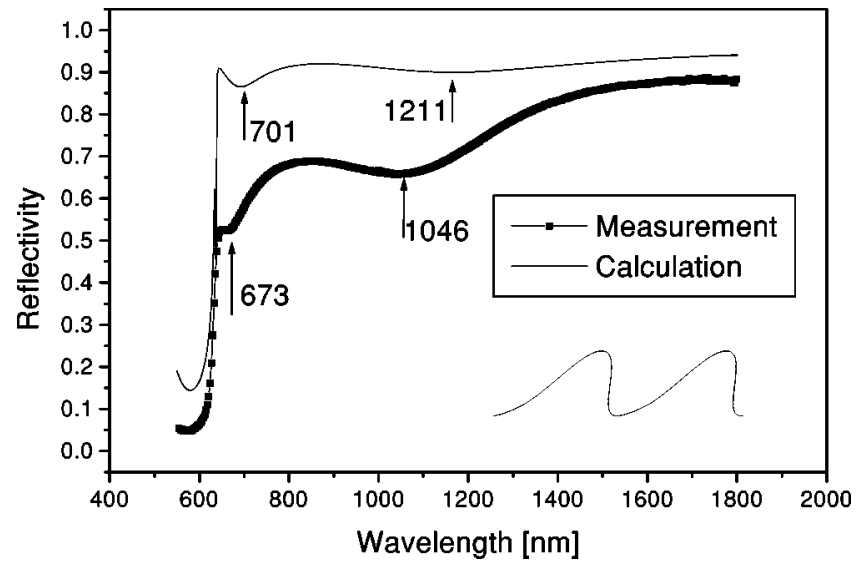

FIG. 15. Wavelength-dependent reflectivity $\left(\theta=4^{\circ}, \psi=90^{\circ}\right)$ of the grating etched at $\theta_{\text {etch }}=30^{\circ}$ as determined from a measurement compared to theory. The surface profile as determined by electron microscopy is shown in the graph.

flectivities with scaling factors between 0.5 and 1 are seen to correspond roughly to the measured data.

With respect to the dispersion of the $\mathrm{MO}_{1}$ resonance, the correspondence between theory and experiment is good. With increasing depth, this feature is slowly shifted into the infrared. The strength of the resonance is not significantly altered for varying amplitudes. Some decrease in strength is predicted for grating amplitudes that are significantly higher than the measured ones. They were not accessible to experimental investigations.

The influence of an increasing grating amplitude on the position and strength of the $\mathrm{ME}_{1}$ resonance is measured as predicted by the calculation: a fast redshift is accompanied by a significant weakening that makes the resonance difficult to see, especially in the calculated data. For this reason, this feature is marked in the graph by open circles. The absolute depth of this minimum is smaller in the calculation than in the experiment.

By choosing an etching angle $\theta_{\text {etch }}=30^{\circ}$, a higher asymmetry is introduced to the grating. The measured and modeled reflectivity of such a structure is presented in Fig. 15. $\mathrm{ME}_{1}$ and $\mathrm{MO}_{1}$ can still be seen and the effect of the increasing asymmetry is consistent between theory and experiment.

$\mathrm{MO}_{1}$ is again observed at a shorter wavelength than predicted. It has become shallower than in the less asymmetric grating. $\mathrm{ME}_{1}$ is observed at a wavelength that is smaller than predicted. It has significantly gained in strength in the measurement. While the absolute strength is still underestimated in the calculations, an increase compared with the calculations of more symmetric structures can be observed.

The measured dispersion of the resonances on this grating as a function of the grating amplitude and the corresponding calculation are shown in Fig. 16. The $\mathrm{MO}_{1}$ resonance is relatively weak and weakens further with increasing amplitude, accompanied by a redshift. For the highest grating amplitude that could be produced, this resonance has died out. Here another reflectivity minimum is observed close to the pseudocritical edge, which is assigned to the $\mathrm{ME}_{2}$ resonance, which has to our knowledge not been observed before. Its

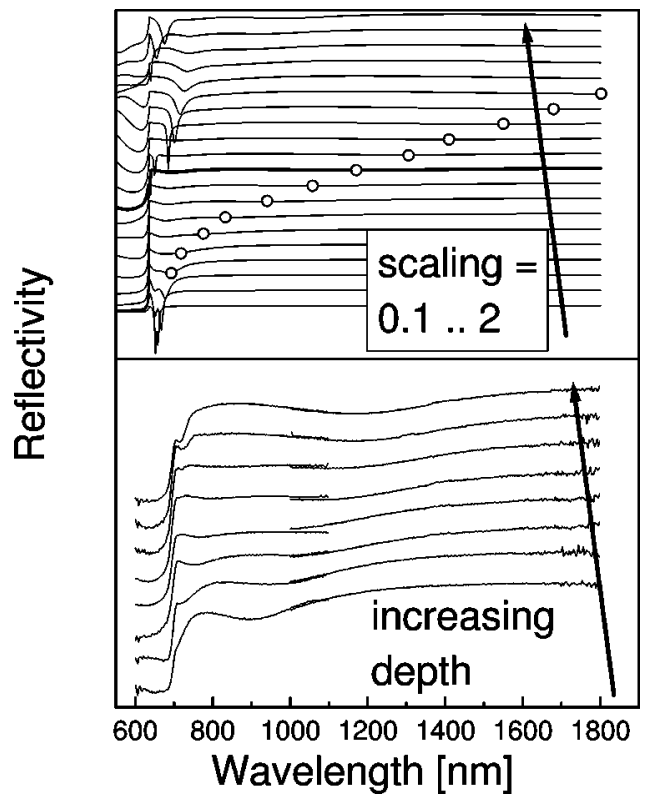

FIG. 16. Modeled (top) and measured (bottom) wavelengthdependent reflectivity $\left(\theta=4^{\circ}, \psi=90^{\circ}\right)$ from a grating etched under $\theta_{\text {etch }}=30^{\circ}$ for different grating amplitudes. The position of the $\mathrm{ME}_{1}$ resonance is indicated by open circles in the calculated data. The thicker line corresponds to the grating shape as determined by electron microscopy.

appearance is predicted for amplitudes that are slightly higher than measured. Thus, the measurements can be roughly assigned to the calculations based on scaling factors between 0.5 and 1.1 .

The $\mathrm{ME}_{1}$ resonance is still showing a redshift with increasing amplitude. Its strength has increased compared to the less blazed grating profiles that were discussed before but it is still much weaker in the theoretical calculation than in the experiment.

Additional experiments were performed on gratings with $\theta_{\text {etch }}=20^{\circ}$ and $\theta_{\text {etch }}=25^{\circ}$, the data do not show qualitatively new features and are therefore not shown. It should be noted though that the $\mathrm{ME}_{2}$ resonance could be observed on these samples as well.

The effects of a further increased asymmetry of the grating profile are presented in theory and experiment in Fig. 17. There are still two resonances visible. But since the grating is already strongly blazed, they cannot be labeled as MO/ME resonances although they seem to evolve from those.

The short-wavelength resonance is significantly shifted towards the pseudocritical edge compared to the grating shapes that were discussed above. In the experiment, this resonance appears smeared out compared to the prediction, this effect can be assigned to the finite experimental resolution in wavelength.

The dispersion of the short-wavelength resonance is different from the less asymmetric grating structures investigated above. In the measurement, the left resonance first moves to shorter wavelengths with increasing amplitude, then it comes back to longer wavelengths when the grating amplitude is further increased. In the calculation, the reso- 


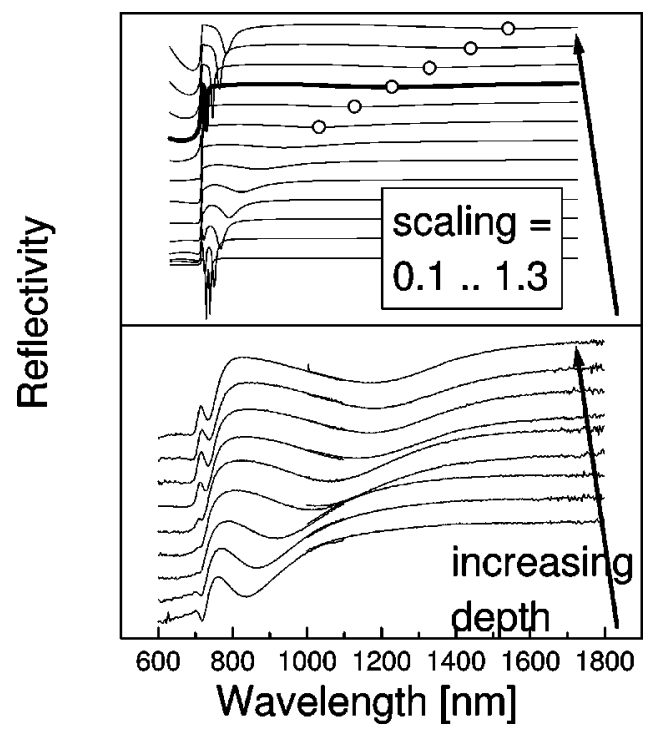

FIG. 17. Modeled (top) and measured (bottom) wavelengthdependent reflectivity $\left(\theta=4^{\circ}, \psi=90^{\circ}\right.$ ) from a grating etched under $\theta_{\text {etch }}=40^{\circ}$ for different grating amplitudes. A weak resonance is marked by open circles where it is hard to see. The thicker line corresponds to the grating shape as determined by electron microscopy.

nance also first moves to the left, but it disappears completely to the left of the pseudocritical angle. With further increasing amplitude, a well-pronounced resonance comes in again from shorter wavelengths, moving quickly to the infrared without disturbance. This suggests that the grating is already blazed so strongly that the original description, as resonances having even and odd symmetry with mutual perturbation at the crossing points, is not appropriate any more. The observed behavior resembles already the reflectivity that is found for strongly overhanging structures (compare Ref. 15).

The long-wavelength resonance is stronger compared to all other profiles that were investigated before. The absolute strength is underestimated in the model calculation.

\section{A stationary resonance}

It was already pointed out by Wanstall et al. ${ }^{15}$ that the wavelength at which resonances are found on very deep and overhanging gratings does not change if the (polar) angle of incidence of the incoming beam is varied in the classical mount. In contrast to this behavior, the excitation of ordinary surface plasmons on shallow diffraction gratings can be described by a matching of the in-plane wave vectors of the surface resonance and a higher diffracted order of the incident light and is strongly angle dependent. ${ }^{1}$ This theoretical prediction is checked here on the sample that was etched using $\theta_{\text {etch }}=40^{\circ}$. The wavelength-dependent reflectivity is shown for polar angles between $4^{\circ}$ and $54^{\circ}$ for both measurement and modeing in Fig. 18. For the calculation, a linear scaling factor of 0.95 was applied to the function representing the grating shape [compare Eq. (2.3)] obtained from

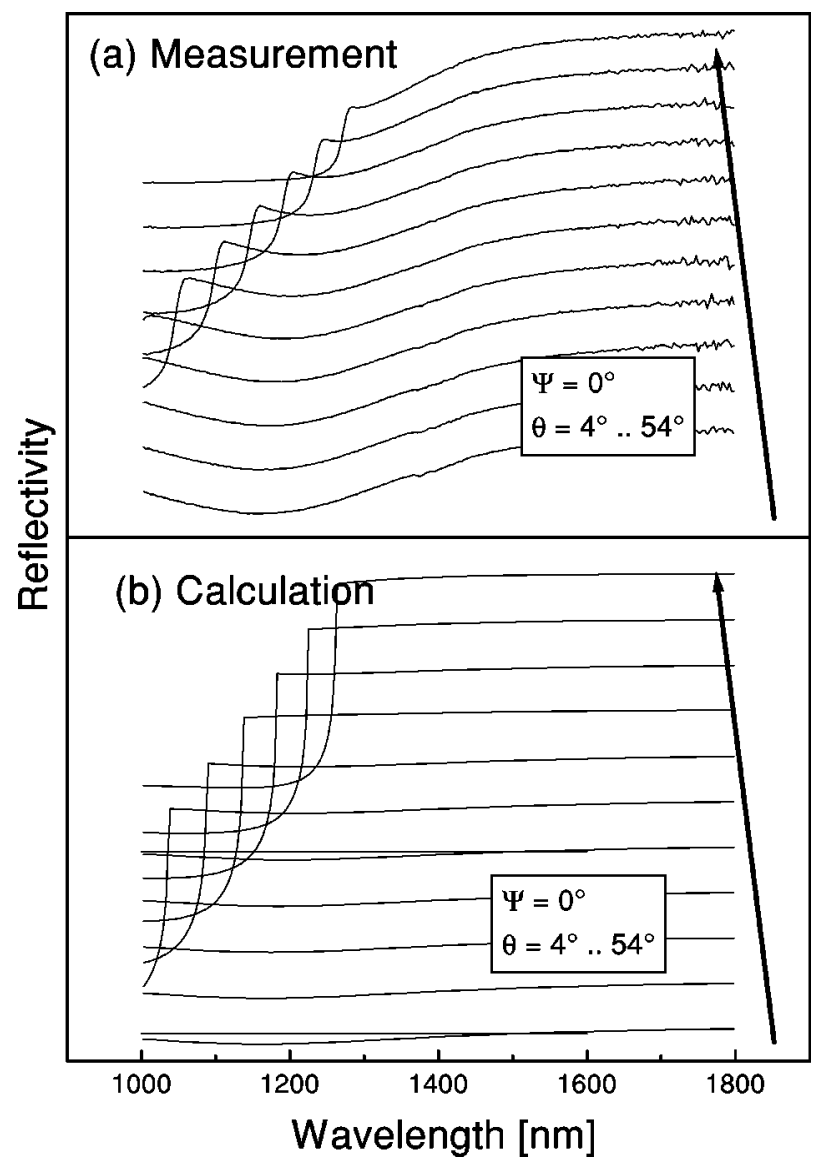

FIG. 18. Modeled (top) and measured (bottom) wavelengthdependent reflectivity of the grating that was etched at $\theta_{\text {etch }}=40^{\circ}$ as a function of wavelength. Different polar angles $\theta$ are chosen for $\psi=0^{\circ}$ (classical mount). The straight horizontal line in (b) is added to the graph as a guide to the eye to see the reflectivity minimum.

the scanning-electron-microscopy image so that the minimum position for $\theta=4^{\circ}$ coincides with that measured $(\lambda$ $=1166 \mathrm{~nm})$.

Good agreement between theory and measurement is observed with respect to the resonance wavelength of the broad minimum, until (at $\theta=54^{\circ}$ ) the pseudocritical edge reaches the resonance and makes its observation impossible. It should be noted that this minimum is, as before, seriously underestimated in theory. The change of resonance wavelength with changing polar angle $\theta$ is far less pronounced than for the "free" surface plasmons on shallow gratings. The small dispersion that is still observable leads to the conclusion that this grating is not a perfect example for a very deep structure in the sense of the paper by Wanstall et al.

\section{The deep symmetric grating}

On the symmetrically etched gratings no deep resonances were observed for wavelength scans at normal incidence. This is shown in Fig. 19 where the wavelength-dependent reflectivity is plotted.

It is clear that the coupling to a resonance with a magnetic field with odd symmetry $\left(\mathrm{MO}_{1}\right)$ in a symmetric configuration is not possible because that would break the symmetry 


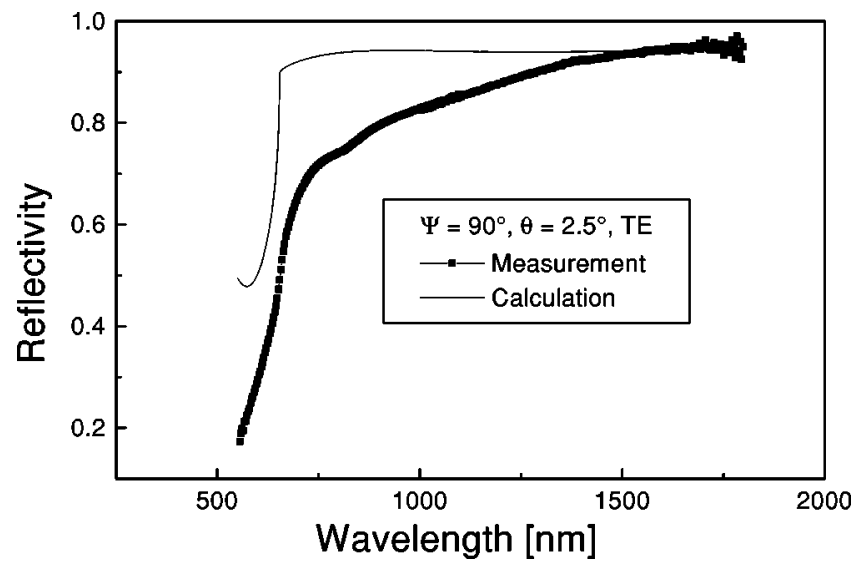

FIG. 19. Wavelength-dependent reflectivity $\left(\theta=4^{\circ}, \psi=90^{\circ}\right)$ of a symmetric grating $(\Lambda=654.5 \mathrm{~nm})$. The dots mark measured points and the straight line is based on theory.

of the initial problem. The very weak minimum at $\lambda$ $=812 \mathrm{~nm}$ in the measurement indicates that the etching process may have been not perfectly symmetric. The absence of the first resonance with even magnetic field cannot be explained by symmetry arguments; for a symmetric broad-peak grating as discussed in Sec. II C it would be visible. The very weak signature of this $\mathrm{ME}_{1}$ indicates that we are dealing with a broad-trough profile. For these calculations, the grating profile that was determined by electron microscopy was slightly modified for the model calculations because the latter one would not have given the right position of the $\mathrm{MO}_{1}$ resonance. However, for the following considerations, the correct description of the position of $\mathrm{MO}_{1}$ with respect to the pseudocritical edge is crucial for a meaningful comparison between theory and experiment.

The possibility of exciting the $\mathrm{MO}_{1}$ resonance on this grating by breaking the symmetry of the reflection geometry via the grating rotation is illustrated by measurement and theory (Fig. 20). It is clearly seen by both experiment and theory that for increasing polar angle $\theta$, a distinct resonance is observed. Here the broken symmetry of the experimental geometry for $\theta \neq 0^{\circ}$ allows for the coupling to the $\mathrm{MO}_{1}$ resonance. It should be noted that the minimum shows a redshift for increasing polar angle $\theta$. Comparison with Fig. 6 shows that this resonance has evolved from the GLRP in the shallow grating limit, which exhibits a blueshift for increasing $\theta$. This observation underlines the interpretation that $\mathrm{MO}_{1}$ is a strongly localized resonance for a broad range of angles of incidence.

\section{E. The photonic mode density on the surface}

In the preceding section, a phenomenon is described that was found in the experiment but not in the calculations. Figure 21 shows the measured reflectivity of a symmetric grating as a function of wavelength in the classical mount $(\psi$ $=0^{\circ}$ ) for different polar angles $\theta$, exceeding the ones that were already investigated in Sec. IV D.

Some rapid changes in the reflectivity with wavelength are observed due to different mechanisms, two of them can

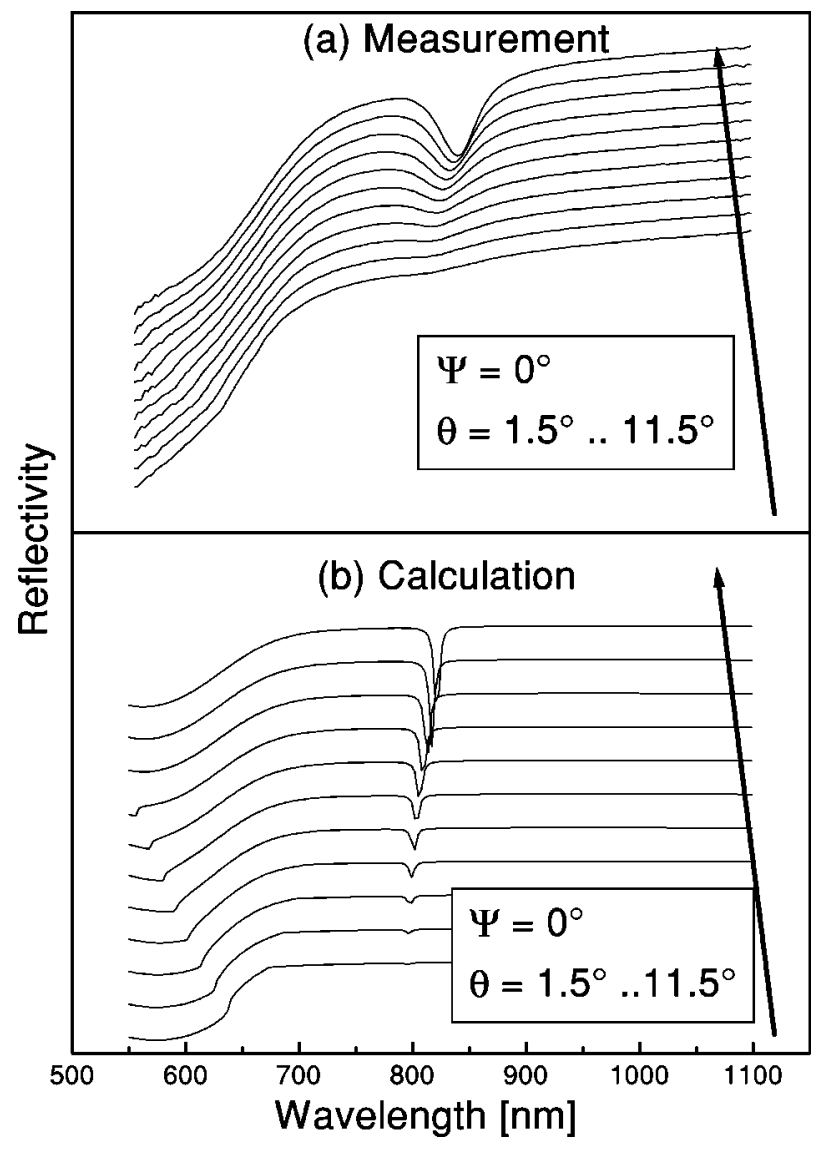

FIG. 20. Measured (top) and modeled (bottom) wavelengthdependent reflectivity (TM polarization) of the symmetric grating $(\Lambda=654.5 \mathrm{~nm})$ for varying polar angle $\theta$ in the classical mount $\left(\psi=0^{\circ}\right)$.

be assigned to resonances that are predicted by theory and do not require further explanation: At $\theta=1.5^{\circ}$, the $\mathrm{MO}_{1}$ resonance is located at $\lambda=812 \mathrm{~nm}$. For increasing polar angle, it gets deeper due to the increasingly broken symmetry and shows a redshift. The pseudocritical edge is found at $\lambda$ $=676 \mathrm{~nm}$ for $\theta=1.5^{\circ}$ and this shifts to the red, too.

Careful inspection of the data reveals an additional feature, which is only seen in the measured data and is completely missing in the theoretical prediction. A kink is observed at $\lambda=820 \mathrm{~nm}$ that is completely independent of the angle of incidence and becomes more pronounced for increasing polar angle. The following explanation for this kink is proposed: The existence of a surface resonance enhances the number of possible electromagnetic waves with a given frequency. A review of the concept of photonic mode density is given by Barnes. ${ }^{23}$ This has the consequence that randomscattering processes become stronger when surface resonances with the appropriate frequencies are supported because new decay channels are established for the photon.

Here, the wavelength where the kink is observed coincides with the shortest possible wavelength where the $\mathrm{MO}_{1}$ resonance may be excited. This leads us to the conclusion that $\mathrm{MO}_{1}$ enhances the photonic mode density for $\lambda$ $>820 \mathrm{~nm}$, which results in an increase in random scattering 


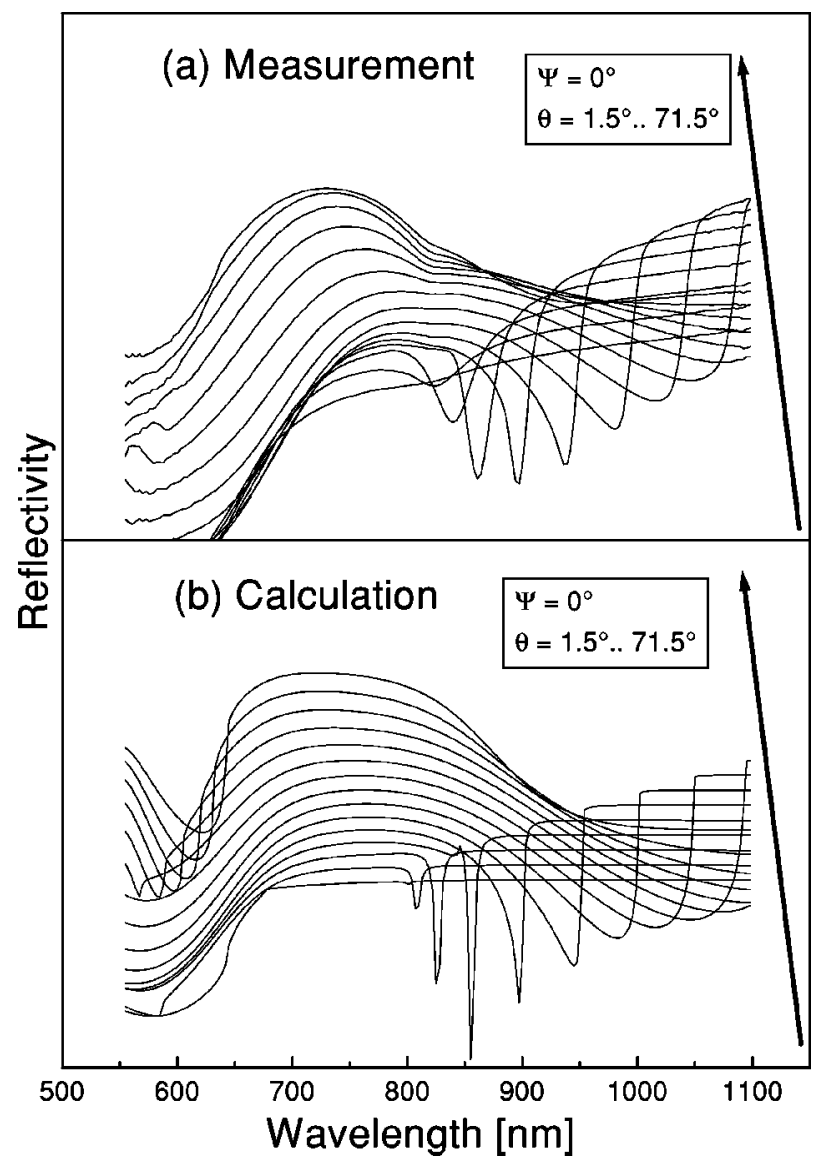

FIG. 21. Wavelength-dependent reflectivity of the symmetric grating $(\Lambda=654.5 \mathrm{~nm})$ in the classical mount $\left(\psi=0^{\circ}\right)$ for polar angles $(\theta)$ between $1.5^{\circ}$ and $71.5^{\circ}$ as obtained from measurement (top) and theory (bottom).

into this surface mode. This effect is responsible for the reduction of the reflected light for wavelengths exceeding $\lambda$ $=820 \mathrm{~nm}$.

\section{CONCLUSION AND OUTLOOK}

The reflectivity of deep gratings with different depths and degrees of asymmetry was investigated. First, an overview of the resonances that are supported by deep gold gratings was given, based on numerical calculations. By considering the symmetry of the electromagnetic-field distributions, the observations could be assigned to two families of resonances. These resonances are characterized by a magnetic field with either even (ME) or odd (MO) symmetry.

On asymmetric gold gratings, two ME resonances and one MO resonance was identified in the experiments and their dispersion and strength was found to be in satisfactory agreement with theory. A theoretical prediction about a strongly decreased impact of the angle of incidence on the resonance wavelength could be experimentally verified.

On symmetric gold gratings, the possible geometries that allow coupling to the $\mathrm{MO}_{1}$ resonance can again be explained by symmetry considerations. Illustrative experimental and theoretical results were presented.

Some other experimental observations were not reflected by the theoretical modeling. Scattering processes may explain the reduced reflectivity close to the pseudocritical edge in all measurements, and the appearance of a stationary kink that was discussed in Sec. IV C, as well as the underestimated strength of the $\mathrm{ME}_{1}$ resonance.

Many questions remain open after these investigations and provide a strong motivation to continue working on deep metallic gratings. The most obvious challenge is the preparation of deeper grating structures in order to see the higherorder members of the two families of resonances. A classification of the possible resonances that is valid not only on symmetric and slightly blazed gratings, but for all possible shapes of the grating surface is still to be developed. Finally, the introduction of scattering processes in the modeling routine may allow one to eliminate the discrepancies between theory and measurement.
${ }^{1}$ H. Raether, Surface Plasmons (Springer, Berlin, 1988).

${ }^{2}$ R. W. Ritchie, E. T. Arakawa, J. J. Cowan, and R. N. Hamm, Phys. Rev. Lett. 21, 1530 (1968).

${ }^{3}$ W. L. Barnes, T. W. Preist, S. C. Kitson, and J. R. Sambles, Phys. Rev. B 54, 6227 (1996).

${ }^{4}$ D. Heitmann, N. Kroo, C. Schulz, and Z. Szentirmay, Phys. Rev. B 35, 2660 (1987).

${ }^{5}$ U. Schröter and D. Heitmann, Phys. Rev. B 60, 4992 (1999).

${ }^{6}$ S. C. Kitson, W. L. Barnes, G. W. Bradberry, and J. R. Sambles, J. Appl. Phys. 79, 7383 (1995).

${ }^{7}$ B. Fischer, T. M. Fischer, and W. Knoll, Appl. Opt. 34, 5773 (1995).

${ }^{8}$ W. L. Barnes, T. W. Preist, S. C. Kitson, J. R. Sambles, N. P. K. Cotter, and D. J. Nash, Phys. Rev. B 51, 6227 (1995).

${ }^{9}$ T. W. Preist, J. B. Harris, N. P. Wanstall, and J. R. Sambles, J. Mod. Opt. 44, 1073 (1997).
${ }^{10}$ J. Chandezon, M. T. Dupuis, G. Cornet, and D. Maystre, J. Opt. Soc. Am. 72, 839 (1982).

${ }^{11}$ B. Laks, D. L. Mills, and A. A. Maradudin, Phys. Rev. B 10, 4965 (1981).

${ }^{12}$ F. J. Garcia-Vidal and J. B. Pendry, Phys. Rev. Lett. 77, 1163 (1996).

${ }^{13}$ T. Lopez-Rios, D. Mendoza, F. J. Garcia-Vidal, J. SanchezDehesa, and B. Pannetier, Phys. Rev. Lett. 81, 665 (1998).

${ }^{14}$ M. B. Sobnack, W. C. Tan, N. P. Wanstall, T. W. Preist, and J. R. Sambles, Phys. Rev. Lett. 80, 5667 (1998).

${ }^{15}$ N. P. Wanstall, T. W. Preist, W. C. Tan, M. B. Sobnack, and J. R. Sambles, J. Opt. Soc. Am. A 15, 2869 (1998).

${ }^{16}$ J. A. Porto, F. J. Garcia-Vidal, and J. B. Pendry, Phys. Rev. Lett. 83, 2845 (1999).

${ }^{17}$ N. W. Ashcroft and N. D. Mermin, Solid State Physics (Saunders College, Philadelphia, 1976). 
${ }^{18}$ D. W. Lynch and W. R. Hunter, Comments on the Optical Constants of Metals and an Introduction to the Data for Several Materials (Academic Press, Boston, 1985).

${ }^{19}$ D. Sarid, Phys. Rev. Lett. 47, 1927 (1981).

${ }^{20}$ R. A. Watts, T. W. Preist, and J. R. Sambles, Phys. Rev. Lett. 79, 3978 (1997).
${ }^{21}$ A. A. Maradudin, A. V. Shchegrov, and T. A. Leskova, Opt. Commun. 135, 352 (1997).

${ }^{22}$ S. Astilean, P. Lalanne, and M. Palmaru, Opt. Commun. 175, 265 (2000).

${ }^{23}$ W. L. Barnes, J. Mod. Opt. 45, 661 (1998). 